\title{
Type-II Optical Parametric Oscillator : a versatile source of quantum correlations and entanglement
}

\author{
Julien Laurat, Thomas Coudreau and Claude Fabre* \\ Laboratoire Kastler Brossel, Université P. et M. Curie, \\ Case 74, 4 Place Jussieu, 75252 Paris cedex 05, France
}

(Dated: July 18, 2018)

\begin{abstract}
Type-II Optical Parametric Oscillators are efficient sources of quadrature squeezed or polarizationsqueezed light, intensity correlated beams, and entangled light. We review here the different levels of quantum correlations and entanglement that are reached in this device, and present some applications.
\end{abstract}

\section{INTRODUCTION}

Quantum correlations play a key role in quantum mechanics, in basic issues such as non-locality or decoherence and also in potential applications such as quantum information processing and computation. The existence of correlations between different physical systems is obviously not a specific property of quantum physics : it is simply the consequence of a former interaction, whatever its character, between the systems submitted to the measurement. Consequently, the observation or prediction of a correlation, even perfect, between the measurements of two variables is not at all a proof of the quantum character of the phenomenon under study. One can find in the literature a great deal of criteria setting a border between the classical and the quantum effects, differing by the definitions of the quantum character of a given physical situation. The purpose of this paper is to review some criteria for quantum correlations (section II) and to describe how a single device, namely a type-II Optical Parametric Oscillator (section $\Pi$ A produces various kinds of correlations fulfilling these criteria (sections IIIB and IIIC). We will also show that the same device can generate entangled states in a non-standard form (section [V]. This last section will provide a good insight into general properties of two-mode gaussian states, illustrated in terms of covariance matrices.

The results presented in this paper are detailed in Refs. 1, 2, 3, 4, 5, 6].

\section{CORRELATION CRITERIA}

Let us consider two light beams denoted by indices 1 and 2. We denote by $\delta X_{1,2}$ one quadrature component of these beams, which can be measured either by direct photodetection (amplitude quadrature) or by an homodyne detection, normalized in such a way that vacuum fluctuations have a variance equal to 1 . We restrict ourselves in this paper to the "balanced" case when the two

\footnotetext{
${ }^{*}$ To whom correspondence should be addressed (fabre@spectro.jussieu.fr)
}

beams have equal variances $F$ on these quadratures, and also equal frequencies. More general criteria in the unbalanced case can be found in [1]. Let us stress also that we are in the case where the quantum properties of the system are well described by a linearized approach of quantum fluctuations.

\section{A. "Gemellity"}

A first criterion of quantum correlations can be defined as follows: the correlation measured in the system cannot be described by a semi-classical model involving classical electromagnetic fields having classical fluctuations.

It is easy to show that the classical character of light fields is preserved by linear passive optical devices, which involve only linear, energy-preserving, optical elements like beamsplitters and free propagation. In order to ascertain the quantum character of correlations existing between $\delta X_{1}$ and $\delta X_{2}$, the simplest way is therefore to process the two beams by all possible linear passive optical devices : if one is able to produce in such a way a beam having fluctuations below the quantum noise limit, that is well-known to be "non-classical", the initial correlation will also be termed as non-classical.

For balanced beams, the best linear processing is simply to send them on a 50/50 beam-splitter: one obtains at one of its output ports a beam with quadrature fluctuations $\delta X_{\text {out }}$ given by

$$
\delta X_{\text {out }}=\frac{\delta X_{1}-\delta X_{2}}{\sqrt{2}}
$$

having a variance given by:

$$
G=\frac{1}{2}\left\langle\left(\delta X_{1}-\delta X_{2}\right)^{2}\right\rangle
$$

The correlation will be said to be non-classical when this quantity, that can be called the "gemellity", is smaller than 1. $G$ can also be written in terms of the noise variance of each beam $F$ and of the normalized correlation coefficient $C_{12}$ :

$$
G=F\left(1-\left|C_{12}\right|\right) .
$$

Therefore a correlation is non-classical when the normalized correlation function fulfills the following condition: 


$$
\left|C_{12}\right|>1-\frac{1}{F}
$$

Thus the larger the classical noise is on each beam, the more stringent the condition becomes.

Finally, let us stress that $G$ can be easily measured experimentally: this is done in all homodyne detection schemes of squeezing, which actually measures the quantum character of the correlation existing between the two beams produced by mixing the field to measure with the local oscillator, and in all twin beams experiments [2, 7].

\section{B. Quantum Non Demolition correlation}

When two observables $M_{1}$ and $M_{2}$ are correlated, the measurement of $M_{2}$ gives some information about the value of $M_{1}$ without any interaction with system 1 . Correlations provide therefore opportunities for Non Demolition measurements. One is led to a second criterion of quantum correlation: the correlation is such that the information extracted from the measurement on one field provides a Quantum Non Demolition measurement of the other [8].

This criterion is related to the conditional variance given by :

$$
V_{1 \mid 2}=F_{1}\left(1-C_{12}^{2}\right) .
$$

where $F_{1}$ is the noise of beam 1 normalized to shot noise. QND correlations correspond to values of $V_{1 \mid 2}$ below 1, and therefore to a correlation satisfying the inequality:

$$
\left|C_{12}\right|>\sqrt{1-\frac{1}{F}}
$$

Eq. [5 can also be expressed in terms of the gemellity:

$$
V_{1 \mid 2}=V_{2 \mid 1}=G\left(1+\left|C_{12}\right|\right)=2 G-\frac{G^{2}}{F} .
$$

It is easy to show from these relations that all QNDcorrelated beams have a gemellity smaller than 1, whereas a gemellity smaller than 0.5 is required to have QND-correlated beams (in the limit of large individual noise).

\section{Inseparability}

Let us now define a new criterion related to entanglement: the correlation cannot be described by separable quantum states. Can the state be written as (a sum of) tensor products or not?

If one is sure that the system is in a pure state, separable or factorizable state vectors give rise to no correlations at all, whatever the observables: the existence of a non-zero correlation, even "classical", on a single quadrature is sufficient to prove the inseparability of the state.

When the state is mixed, which is the general case, this is no longer the case. Let us consider for example the mixed state described by the density matrix

$$
\rho=\sum_{n} p_{n}(|1: n\rangle \otimes|2: n\rangle)(\langle 1: n| \otimes\langle 2: n|)
$$

where $|1,2: n\rangle$ is a Fock state with $n$ photons in mode 1,2 . This highly non-classical state has perfect intensity correlations $\left(C_{12}=1\right)$, so that $\left.G=V_{1 \mid 2}=0\right)$. However, it is a separable state, being a statistical mixture of factorized state vectors.

In order to ascertain the separable character of the physical state of a system, one needs to make two joint correlation measurements on non-commuting observables on the system, and not only one, as was the case in the two previous criteria. More precisely, Duan et al. [9] have shown that, in the case of Gaussian states for which the covariance matrix is expressed in the so-called standard form, there exists a necessary and sufficient criterion of separability in terms of the quantity $\mathcal{I}$, that we will call separability, and is given by :

$$
\begin{aligned}
\mathcal{I} & =\frac{1}{4}\left(\left\langle\left(\delta X_{1}-\delta X_{2}\right)^{2}\right\rangle+\left\langle\left(\delta P_{1}+\delta P_{2}\right)^{2}\right\rangle\right) \\
& =\frac{1}{2}\left(G_{X}+G_{P}\right) .
\end{aligned}
$$

The separability appears as the half-sum of the gemellity measuring the correlations between quadratures $\delta X$ and the (anti)gemellity measuring the anticorrelations between $\delta P$. A state for which $\mathcal{I}$ is smaller than one will be a non-separable or entangled state. As a consequence, classically correlated beams, for which these two gemellities are larger than 1, are separable.

Let us note that in the case of symmetric gaussian states the entanglement can be quantified by a quantity called entropy of formation - or entanglement of formation $E O F$-, that was introduced in Ref. 10]. It represents the amount of pure state entanglement needed to prepare the entangled state. This quantity is related to the value of the inseparability $\mathcal{I}$ by:

$$
E O F=c_{+} \log _{2}\left(c_{+}\right)-c_{-} \log _{2}\left(c_{-}\right)
$$

with

$$
c_{ \pm}=\left(\mathcal{I}^{-1 / 2} \pm \mathcal{I}^{1 / 2}\right)^{2} / 4
$$

EOF takes a positive value only for entangled beams. Its interest is that it constitutes a real measure of the amount of entanglement. In addition, it is also used in the discrete variable regime.

A more general quantity has been introduced to characterize the entanglement: the logarithmic negativity [1]. This quantity can be calculated for any arbitrary bipartite system. We will consider it in more detail in the last section of this paper where the generated two-mode state is not in a standard form. 


\section{Einstein-Podolsky-Rosen correlations}

Two correlations give the opportunity of Non Demolition measurements on two non-commuting variables. As for a single quadrature, one can be interested in the quality of the information that one gets on one beam by measuring the other. This question is related to the question asked by Einstein, Podolsky and Rosen in their famous paper [12]. In particular, we will say that we have "EPR beams" when the information extracted from the the measurement of the two quadratures of one field provide values for the quadratures of the other which "violate" the Heisenberg inequality. This criterion was considered and discussed extensively by Reid and co-workers 13. They showed that to characterize this property, one can use the product of the conditional variances,

$$
\mathcal{V}=V_{X_{1} \mid X_{2}} \cdot V_{P_{1} \mid P_{2}}
$$

When this quantity is smaller than one we will say that we have "EPR-correlated beams". Let us note that when this condition is fulfilled, one can perform double QNDmeasurements, that is two QND-measurements on noncommuting quadratures. One can show that all EPRcorrelated beams are not separable, whereas the reverse is not true. EPR-correlation is therefore the strongest of the correlation criteria that we have listed here. One can envision other criteria which are even stronger, but not relevant for the problem of measuring correlated quadratures with Gaussian statistics that we are considering here.

\section{EXPERIMENTAL INVESTIGATION OF QUANTUM CORRELATIONS}

In this section, we will show how these various criteria can be tested using the states produced by a triply resonant type-II Optical Parametric Oscillator. Such a system consists of a triply-resonant optical cavity containing a type-II phase matched $\chi^{(2)}$ crystal. Spontaneous parametric down-conversion which occurs in such crystals is well known to produce twin photons, that is photons created in pairs. When such a crystal is placed inside a cavity and the system pumped above a critical value (threshold), one generates intense beams which are correlated. The system transfers the correlations existing in the discrete regime to the continuous variable one. The $\mathrm{OPO}$ is thus an ideal system to test the various criteria that we have mentioned above.

\section{A. Experimental set-up}

The experimental setup is shown in Fig. A continuous-wave frequency-doubled Nd:YAG laser pumps a triply resonant OPO above threshold, made of a semi-monolithic linear cavity. The intensity reflection

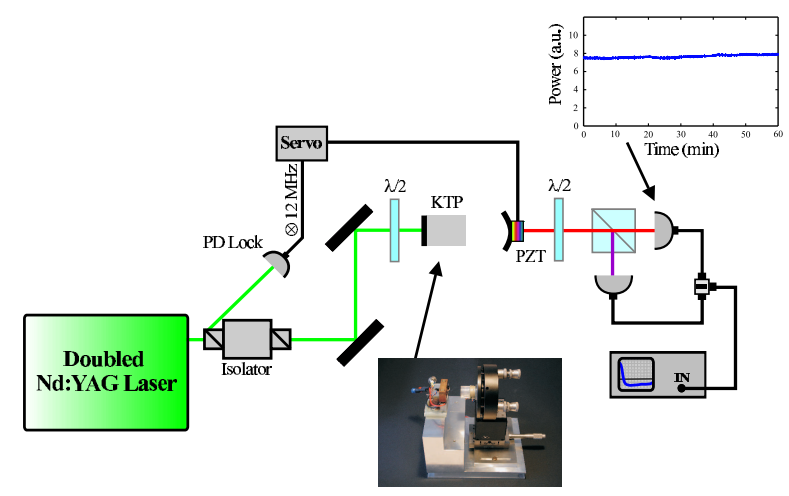

FIG. 1: A cw doubled Nd:YAG laser pumps above threshold a type-II OPO. Intensity correlations are directly measured by a balanced detection scheme. PD Lock: FND-100 photodiode for locking of the OPO.

coefficients for the input coupler are $95 \%$ for the pump at $532 \mathrm{~nm}$ and almost $100 \%$ for the signal and idler beams at $1064 \mathrm{~nm}$. The output mirror is highly reflective for the pump and its transmission coefficient $T$ can be chosen to be 5 or $10 \%$. With $T=5 \%$, at exact triple resonance, the oscillation threshold is less than $15 \mathrm{~mW}$. In spite of the triple resonance which generally makes OPOs much more sensitive to disturbances, length and temperature controls enable stable operation over more than one hour without mode-hopping.

\section{B. $" 2 \times 1$ quadrature" case}

\section{Twin beams}

Type II optical parametric oscillators are well-known to generate above threshold highly quantum correlated bright twin beams. Intensity correlations were experimentally observed several years ago and applied to measurements of weak physical effects [7]. We describe here a recent improvement of the observed correlation.

Intensity correlations are directly measured by a balanced detection scheme (Fig. 1). The signal and idler orthogonally polarized beams are separated on a polarizing beam splitter and detected on a pair of high quantum efficiency photodiodes. With a transmission $T=10 \%$ for the output mirror, we have obtained a noise reduction of $9.7 \pm 0.5 \mathrm{~dB}(89 \%)$ around $5 \mathrm{MHz}$ (Fig. 2), which corresponds to a gemellity of $G=0.11$. To the best of our knowledge, this noise reduction is the strongest reported to date in the experimental quantum optics field.

\section{QND correlations and conditional preparation of a non-classical state}

The observed correlation is strong enough to yield a conditional variance well below 1 . We will show now 


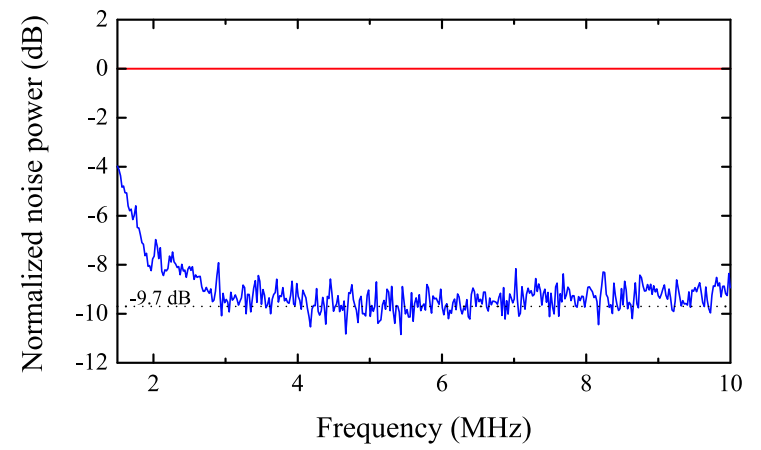

FIG. 2: Normalized noise power of the intensity difference of the signal and idler as a function of the frequency, after correction of the electronic noise.

that such a QND-correlation can be used to produce a squeezed state via conditional preparation performed on continuous variables.

A well-known technique to generate a single photon state from twin photons is to use the method of conditional measurement: if one labels (1) and (2) the two modes in which the twin photons are emitted, it consists in retaining in the information collected in mode (1) only the counts occurring when a photon is detected in mode (2) within a given time window $\Delta T$. State preparation by conditional measurement can be readily extended to the continuous variable regime, where the instantaneous values of the signal and idler photocurrents play the role of the occurrence of counts in the photon counting regime. The technique consists in selecting the signal photocurrent $I_{s}$ only during the time intervals when the idler intensity $I_{i}$ has a given value $I_{0}$ (within a band $\Delta I$ smaller than the photocurrent standard deviation). The measurements outside these time intervals are discarded. If the correlation is perfect and the interval $\Delta I$ close to zero, the recorded signal intensity is perfectly constant, and an intense number state is generated; in a real experiment, the correlation between the signal and idler photocurrents is not perfect, and the selection band $\Delta I$ is finite, so that the method will not prepare a perfect number state, but a sub-Poissonian state instead.

A theoretical analysis of this protocol [4] shows that in the limit where $\Delta I$ is very small the conditional measurement produces a beam characterized by a Fano factor equal to the conditional variance of the signal and idler beams. This means that the present protocol produces a sub-Poissonian beam when the signal and idler beams are QND-correlated. As shown in Eq. 7] in the limit of large correlations, the residual intensity noise $F$ on the conditionally prepared state will be equal to twice the gemellity $(F=V \simeq 2 G)$.

Figure 3 sums up the experimental results. The Fano factor $F$ of both the signal and idler beams exceeds 100 (20 dB above the shot noise level), and the measured gemellity $G$ is equal to 0.18 ( 0.14 after correction of dark noise). The ensemble of values of the signal intensity for which the idler intensity falls within the selection band
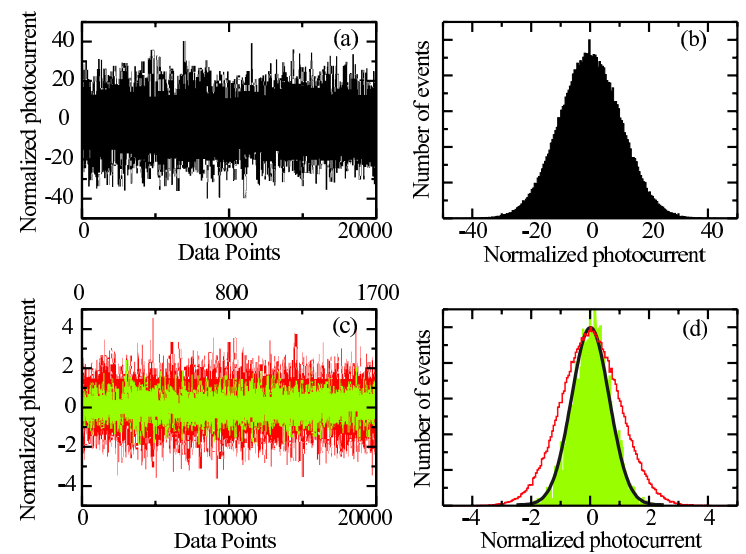

FIG. 3: Experimental results: (a) Idler intensity fluctuations: 200000 acquired points at $3.5 \mathrm{MHz}$ demodulation frequency (only 20000 shown). (b) Corresponding probability distribution. The unit is the width $\sigma_{0}$ of the Poisson distribution of same mean intensity (shot noise). (c) Values of the signal intensity conditionally selected by the value of the idler intensity recorded at the same time (selection bandwidth $\Delta I$ equal to $0.1 \sigma_{0}$ around the mean), superimposed to the corresponding experimentally measured shot noise. (d) Corresponding probability distribution, compared to the Poisson distribution (grey line), displaying the sub-Poissonian character of the conditionally generated state. The black line is a gaussian fit of the intensity distribution.

is given in figure 3 (c): one indeed observes a significant narrowing of the probability distribution below the shot noise level. With a selection bandwidth $\Delta I$ equal to 0.1 times the standard deviation $\sigma_{0}$ of a coherent state having the same power (shot noise level), the conditionally prepared light state has a measured Fano factor $F=0.36$, which turns out to be equal, as expected, to the conditional variance of the twin beams. The success rate of the conditional preparation is around $0.85 \%$ (1700 points out of 200000 are accepted). An advantage of the conditional preparation using continuous variables is that one can use at the same time different selection non-overlapping bands on the idler beam. Each allows one to conditionally prepare a different sub-Poissonian state, each having a Fano factor $F=0.36$. With 200 different selection bands, the overall success rate is close to $100 \%$.

\section{C. $" 2 \times 2 "$ quadratures case \\ 1. Entanglement below threshold}

Type-II OPO below threshold are well-known to generate entangled beams. The first experimental demonstration of EPR correlations in the continuous variable regime in 1992 was performed with such a device 14]. Our experimental setup is similar to the previous one (Fig. 11) but the detection system is now based on two 


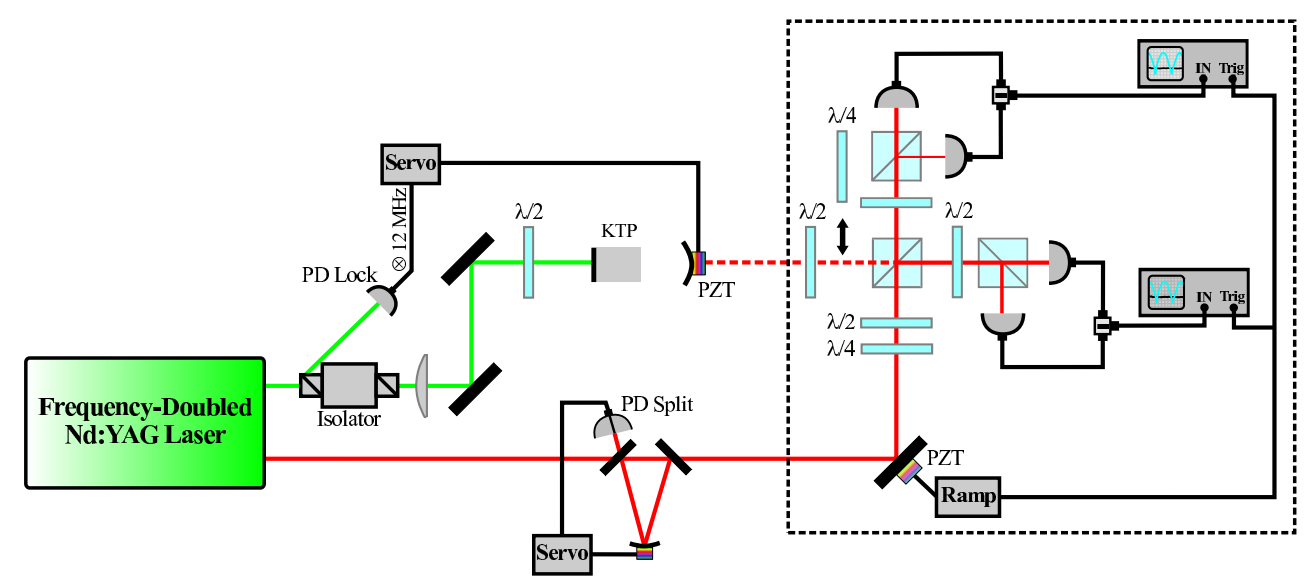

FIG. 4: A doubled Nd:YAG laser pumps a type II OPO, below or above threshold. The generated two-mode state is characterized by two simultaneous homodyne detections. PD Split: split two-element InGaAs photodiode for tilt-locking of the filtering cavity.

simultaneous homodyne detections (Fig. 4). In order to measure the separability $\mathcal{I}$, one must characterize the noise of the superposition modes oriented $\pm 45^{\circ}$ from the axes of the crystal:

$$
A_{+}=\frac{A_{1}+A_{2}}{\sqrt{2}} \quad \text { and } \quad A_{-}=\frac{A_{1}-A_{2}}{\sqrt{2}}
$$

Eq. 9 shows that the signal and idler fields are entangled as soon as these two modes have squeezed fluctuations on orthogonal quadratures. The orthogonally polarized modes are separated on a first polarizing beam splitter at the output of the OPO. A half-wave plate inserted before this polarizing beam splitter enables us to choose the fields to characterize: the signal and idler modes which are entangled, or the $\pm 45^{\circ}$ rotated modes which are squeezed. The detection setup is able to characterize simultaneously the two chosen modes with the same phase reference, and to measure the noise reductions either in quadrature ("in phase homodyne detection") or in phase ("in quadrature homodyne detection"), by inserting or not a $\lambda / 4$ plate in the beam exiting the OPO. This configuration permits a direct and instantaneous verification of the inseparability criterion by simply adding the two squeezed variances.

Typical spectrum analyzer traces while scanning the local oscillator phase are shown on Fig. 5 Normalized noise variances of the $\pm 45^{\circ}$ vacuum modes at a given noise frequency of $3.5 \mathrm{MHz}$ are superimposed for in-phase and in-quadrature homodyne detections. One indeed observes, as expected, correlations and anti-correlations of the emitted modes on orthogonal quadratures. The homodyne detection can be locked on the squeezed quadrature (Figure 5). The observed amount of simultaneous squeezing for the two rotated modes is $-4.3 \pm 0.3 \mathrm{~dB}$ and $-4.5 \pm 0.3 \mathrm{~dB}$ below the standard quantum limit $(-4.7 \pm 0.3 \mathrm{~dB}$ and $-4.9 \pm 0.3 \mathrm{~dB}$ after correction of the electronic noise). This gives a value of the separability of $\mathcal{I}=0.33 \pm 0.02$, well below the unit limit for inseparability. With a measured value of the parameter $F$ of 6.6 , one obtains a product of conditional variances of $0.42 \pm 0.05$, well below 1 , which establishes the EPR character of the measured correlations. The entanglement of formation EOF of the two beams is, according to formulae (10) and (11), equal to $1.1 \pm 0.1$ ebits. To the best of our knowledge, this setup generates the best $\mathrm{EPR} /$ entangled beams to date produced in the continuous variable regime.
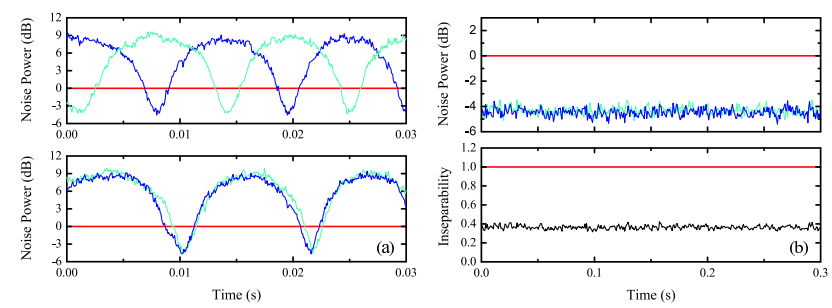

FIG. 5: (a) Normalized noise variances at $3.5 \mathrm{MHz}$ of the $\pm 45^{\circ}$ modes while scanning the local oscillator phase. The first plot corresponds to in-phase homodyne detections and the second one in-quadrature. Squeezing is well observed on orthogonal quadratures. (RBW $100 \mathrm{kHz}, \mathrm{VBW} 1 \mathrm{kHz}$ ) (b) Normalized noise variances at $3.5 \mathrm{MHz}$ of the $\pm 45^{\circ}$ modes and inseparability $\mathcal{I}$ for signal and idler modes. The homodyne detections are in-quadrature and locked on the squeezed quadratures. After correction of the electronic noise, the inseparability criterion reaches $0.33 \pm 0.02$. (RBW $100 \mathrm{kHz}$, VBW $300 \mathrm{~Hz}$ ).

Non-classical properties are generally measured in the $\mathrm{MHz}$ range of Fourier frequencies, because of the presence of large classical noise at lower frequencies. In the present device significant quantum correlations and EPR entanglement have been observed in from $50 \mathrm{kHz}$ to 10 $\mathrm{MHz}$. Figure 6 gives the squeezed variances for low noise frequencies, between $40 \mathrm{kHz}$ and $150 \mathrm{kHz}$. Let us mention that squeezing from a single type-I OPA was recently reported at a record Fourier frequency of $200 \mathrm{~Hz}$ [15]. 


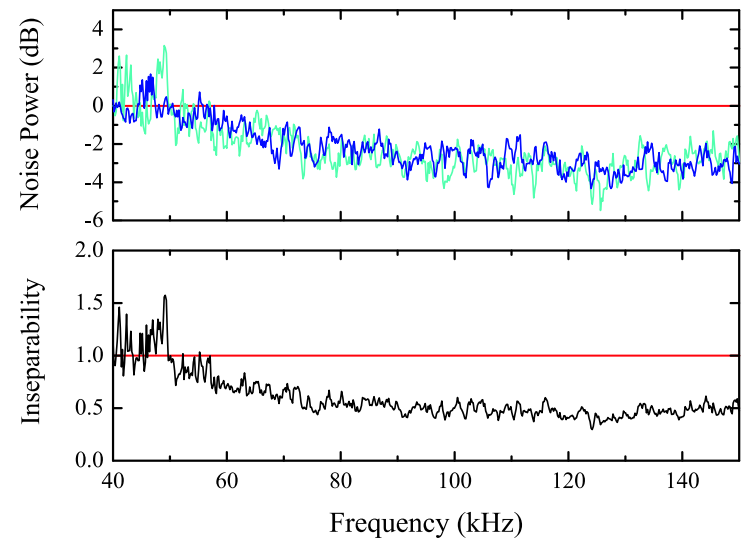

FIG. 6: Normalized noise variances from $40 \mathrm{kHz}$ to $150 \mathrm{kHz}$ of the $\pm 45^{\circ}$ modes after correction of the electronic noise and inseparability criterion for signal and idler modes. Squeezing and entanglement are observed down to $50 \mathrm{kHz}$. (RBW 3 $\mathrm{kHz}, \mathrm{VBW} 10 \mathrm{~Hz}$ )

\section{Bright EPR beams above threshold and polarization squeezing}

A type-II OPO pumped above threshold has been theoretically predicted to be a very efficient source of bright entangled and EPR beams. This means that, in addition to the already demonstrated intensity correlations, phase anticorrelations exist in the system. However, they can be easily measured by usual homodyne detection techniques only in the frequency-degenerate regime. Frequency degeneracy occurs only accidentally above threshold because it corresponds to a single point in the experimental parameter space. Actually, up to now, no direct evidence of such phase anti-correlations has been observed. In 1998, Mason and Wong proposed an elegant way to achieve frequency degenerate operation above threshold [16, 17]: they inserted inside the OPO cavity a birefringent plate making an angle with the axis of the non-linear crystal. The induced linear coupling between the signal and idler results in a locking phenomenon [18]. It has been shown theoretically that in such a "self-phaselocked" OPO the quantum correlations are preserved for small angles of the plate and that the system produces entangled states in a wide range of parameters 19, 20].

In the experiment, the frequency locking phenomenon can be maintained during more than hour. Degenerate operation is confirmed by the fact that the generated mode has now a fixed polarization: at the minimum threshold point, the generated state is linearly polarized at $+45^{\circ}$. Due to the defined phase relation existing now between the signal and idler fields, $A_{+}$is a bright mode, and $A_{-}$has a zero mean value. Figure $\mathbf{7}$ (a) gives the noise power of the mode $A_{-}$while scanning the local oscillator phase, for a transmission $T=5 \%$ and a plate angle of $0.1^{\circ}$. A noise reduction of $4.5 \mathrm{~dB}$ is observed. This strong noise reduction on the mode $A_{-}$confirms the quantum intensity correlation between the signal and
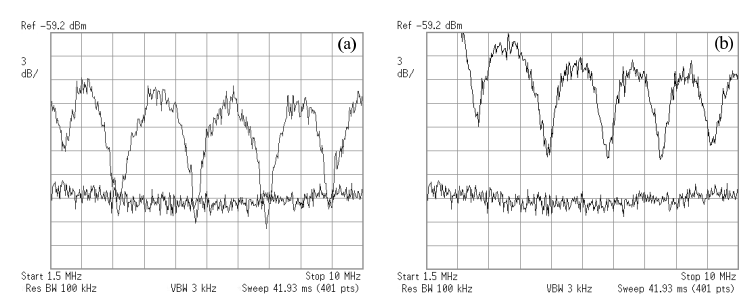

FIG. 7: (a) Noise power of the mode $A_{-}$while scanning simultaneously the phase of the local oscillator and the noise frequency between 1.5 and $10 \mathrm{MHz}$. The lower trace gives the shot noise level. (b) Noise power of the mode $A_{+}$while scanning the phase of the local oscillator, for a noise frequency between 1.5 and $10 \mathrm{MHz}$. The shot noise level is given by the lower trace plus $3 \mathrm{~dB}$.

idler modes. Figure $\mathbf{7}$ (b) shows the noise power of the mode $A_{+}$in the same condition. As the plate angle is very small, a similar amount of noise reduction is expected. However, a slight excess noise of $3 \mathrm{~dB}$ is measured for the minimal noise quadrature: the phase anticorrelations appear to be slightly degraded, probably by external noise sources.

Despite this slight excess noise which prevents from reaching the proof of entanglement in the OPO above threshold, the generated state turns out to be squeezed in the polarization orthogonal to the mean field: $A_{+}$ is the main mode and $A_{-}$the squeezed vacuum one. This condition is required to obtain a so-called "polarization squeezed" state [21, 22, 23]. $4.5 \mathrm{~dB}$ of polarization squeezing has been thus generated in the self-phaselocked OPO. Such states have recently raised great interest, in particular because of the possibility to map quantum polarization state of light onto an atomic ensemble [24].

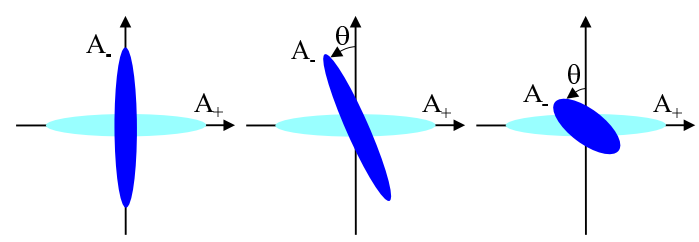

FIG. 8: Fresnel representation of the noise ellipse of the $\pm 45^{\circ}$ rotated modes when the plate angle is increased. Without coupling, squeezing is predicted on orthogonal quadratures. The noise ellipse of the $-45^{\circ}$ mode rotates and the noise reduction is degraded when the coupling increases while the $+45^{\circ}$ rotated mode is not affected.

\section{MANIPULATING ENTANGLEMENT WITH POLARIZATION ELEMENTS}

The self-phase-locked OPO can also be operated below threshold. It produces a two-mode state with strong quantum features which manifest themselves in terms of noise reduction properties in a given polarization basis, 


$$
\begin{gathered}
\Gamma_{A_{1} A_{2}}=\left(\begin{array}{cc|cc}
181.192 & 0 & 179.808 & -0.255 \\
0 & 0.386 & -0.255 & -0.383 \\
\hline 179.808 & -0.255 & 181.192 & 0 \\
-0.255 & -0.383 & 0 & 0.386
\end{array}\right) \Longrightarrow \Gamma_{A_{1} A_{2}}^{\prime}=\left(\begin{array}{cc|cc}
180.839 & 0 & 180.161 & 0 \\
0 & 0.739 & 0 & -0.736 \\
\hline 180.161 & 0 & 180.839 & 0 \\
0 & -0.736 & 0 & 0.739
\end{array}\right) \\
\Gamma_{A_{+} A_{-}}=\left(\begin{array}{cc|cc}
361 & 0 & 0 & 0 \\
0 & 0.00277 & 0 & 0 \\
\hline 0 & 0 & 1.383 & -0.256 \\
0 & 0 & -0.256 & 0.770
\end{array}\right) \Longrightarrow \Gamma_{A_{+} A_{-}}^{\prime}=\left(\begin{array}{cc|cc}
361 & 0 & 0 & 0 \\
0 & 0.00277 & 0 & 0 \\
\hline 0 & 0 & 0.677 & 0 \\
0 & 0 & 0 & 1.476
\end{array}\right)
\end{gathered}
$$

FIG. 9: Numerical example of covariance matrix of the $A_{1} / A_{2}$ modes and the $A_{+} / A_{-}$modes before and after the non-local operation for a plate angle of $\rho=1.3^{\circ} .(\sigma=0.9$ and $\Omega=0)$

and in terms of entanglement and EPR correlations in another. This last section is devoted to the general study of this two-mode Gaussian quantum state.

\section{A. Manipulation of entanglement in the two-mode state produced by the type-II OPO with mode coupling}

In a standard OPO the correlated quadratures are orthogonal to the anti-correlated ones which results in squeezing of the rotated modes on orthogonal quadratures. It is no more the case when a linear coupling is introduced. When the plate angle increases, the correlated quadratures rotate and the correlations are degraded. The evolution is depicted in Fig. 8 through the noise ellipses of the rotated (squeezed) modes. In order to maximize the entanglement between the signal and idler modes, the optimal quadratures have to be made orthogonal [25]. Such an operation consists of a phase-shift of $A_{-}$relative to $A_{+}$. This transformation is passive and "non-local" in the sense of the EPR argument: it acts simultaneously on the two considered sub-systems. In the type II OPO, such "non-local" transformations are easy to perform by inserting polarizing birefringent elements in the total beam, because the two polarization modes are produced by the OPO in the same transverse spatial mode.

As the generated two-mode state is not in the standard form, we need to use a general measure of entanglement. Let us introduce the covariance matrix formalism and the logarithmic negativity. In a given mode basis, the quantum properties of the generated state, of zero mean value, are completely contained in the covariance matrix $\Gamma_{A B}$ defined as:

$$
\Gamma_{A B}=\left(\begin{array}{cc}
\gamma_{A} & \sigma_{A B} \\
\sigma_{A B}^{T} & \gamma_{B}
\end{array}\right)
$$

$\gamma_{A}$ and $\gamma_{B}$ are the covariance matrix of the individual modes while $\sigma_{A B}$ describes the intermodal correlations. The elements of the covariance matrix are written $\Gamma_{i j}=\left\langle\delta R_{i} \delta R_{j}+\delta R_{j} \delta R_{i}\right\rangle / 2$ where $R_{\{i, i=1, \ldots, 4\}}=$ $\left\{X_{A}, Y_{A}, X_{B}, Y_{B}\right\} . X$ and $Y$ corresponds to an arbitrary orthogonal basis of quadratures. In order to measure the degree of entanglement of Gaussian states, a simple computable formula of the logarithmic negativity $E_{\mathcal{N}}$ has been obtained 11] (see also 26] for a general overview). $E_{\mathcal{N}}$ can be easily evaluated from the largest positive symplectic eigenvalue $\xi$ of the covariance matrix which can be obtained from

$$
\xi^{2}=\frac{1}{2}\left(D-\sqrt{D^{2}-4 \operatorname{det} \Gamma_{A B}}\right)
$$

with

$$
D=\operatorname{det} \gamma_{A}+\operatorname{det} \gamma_{B}-2 \operatorname{det} \sigma_{A B}
$$

The two-mode state is entangled if and only if $\xi<1$. The logarithmic negativity can thus be expressed by $E_{\mathcal{N}}=-\log _{2}(\xi)$. The maximal entanglement which can be extracted from a given two-mode state by passive operations is related to the two smallest eigenvalues of $\Gamma$, $\lambda_{1}$ and $\lambda_{2}$, by $E_{\mathcal{N}}^{\max }=-\log _{2}\left(\lambda_{1} \lambda_{2}\right) / 2$ [25].

We give here a numerical example for realistic experimental values $\rho=1.3^{\circ}, \sigma=0.9$ and $\Omega=0$, where $\rho$ stands for the plate angle, $\sigma$ the pump power normalized to the threshold and $\Omega$ the noise frequency. The covariance matrices for the $A_{1} / A_{2}$ modes and for the $A_{+} / A_{-}$ modes are given in Fig. 9 with and without the phaseshift. The matrix of the $A_{+} / A_{-}$modes are well-suited to understand the behavior of the device. At first, the intermodal blocks are zero, showing that these two modes are not at all correlated and consequently are the most squeezed modes of the system: there is no way to extract more squeezing. But one can also note that the diagonal blocks are not diagonalized simultaneously. This corresponds to the tilt angle $\theta$ of the squeezed quadrature of $A_{-}$. A phase-shift of the angle $\theta$ permits to diagonalize simultaneously the two blocks and to obtain squeezing on orthogonal quadratures. The logarithmic negativity $E_{\mathcal{N}}$ has increased in the transformation from 4.06 to 4.53 . The maximal entanglement available has been extracted in this way as $E_{\mathcal{N}}^{\max }=-\log _{2}\left(\lambda_{1} \lambda_{2}\right) / 2=4.53$. 


\section{B. Experimental optimization of entanglement}

Let us now describe how to experimentally optimize the EPR entanglement generated by the self-phase-locked OPO below threshold.

In order to extract the maximal entanglement, one must perform an appropriate phase-shift on the rotated modes. This is achieved by using an association of one $\lambda / 2$ and one $\lambda / 4$ plates added at the output of the OPO. The double homodyne detection we have developed is necessary in order to be able to characterize simultaneously the two modes with the same phase reference.
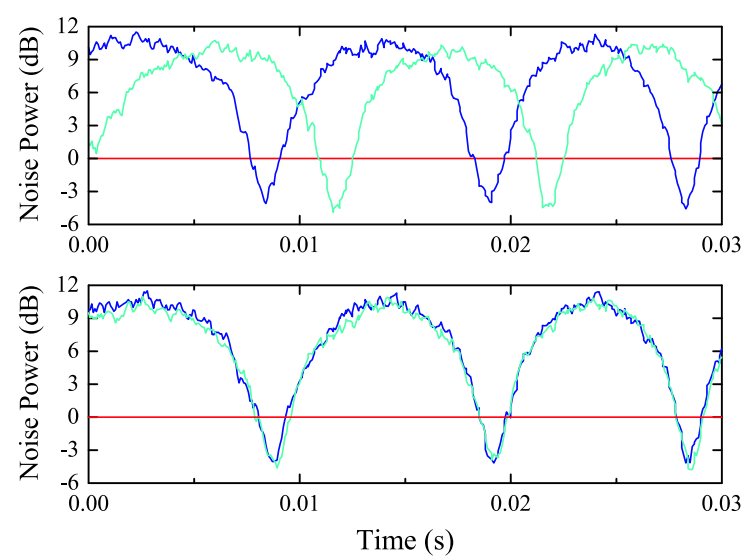

FIG. 10: Normalized noise variances at $3.5 \mathrm{MHz}$ of the rotated modes while scanning the local oscillator phase for a plate angle $\rho=0.3^{\circ}$, before and after the non-local operation. The homodyne detections are in-quadrature. After this operation, squeezing is observed on orthogonal quadratures.
Figure 10] displays the normalized noise variances of the rotated modes for a plate angle of $\rho=0.3^{\circ}$, before and after the phase-shift. The homodyne detections are operated in quadrature so that squeezing on orthogonal quadratures is observed simultaneously on the spectrum analyzers. After the operation is performed, squeezing is obtained on orthogonal quadratures as in a standard type-II OPO without mode coupling. Experimentally, the logarithmic negativity goes from 1.13 to 1.32 , showing that we are able to extract more quantum resource from the state after the operation.

\section{CONCLUSION}

We have seen that type II triply resonant OPO produce in a very stable way the strongest intensity correlation and EPR entanglement to date. Phase-locked, frequency degenerate operation can be obtained using an intracavity birefringent plate. This locking permits the experimental realization of homodyne detection of the quadratures even when the system is operated above threshold. This result opens a very promising way to the direct generation of intense entangled beams and offers a new and simple method to achieve strong polarization squeezing. Below threshold, the self-phase-locked OPO exhibits a very rich and interesting behavior which provides a good insight into entanglement manipulation by passive operations. This opens the way to the manipulation and optimization of quantum properties in highly multimode Hilbert spaces.
[1] N. Treps, C. Fabre, Laser Physics 15, 187 (2005)

[2] J. Laurat, L. Longchambon, T. Coudreau, C. Fabre, Opt. Lett 30, 1177 (2005)

[3] J. Laurat, T. Coudreau, N. Treps, A. Maître, C. Fabre, Phys. Rev. Lett. 91, 213601 (2003)

[4] J. Laurat, T. Coudreau, N. Treps, A. Maitre, C. Fabre, Phys. Rev. A, 69, 33808 (2004)

[5] J. Laurat, T. Coudreau, G. Keller, N. Treps, C. Fabre, Phys. Rev. A 70, 042315 (2004)

[6] J. Laurat, T. Coudreau, G. Keller, N. Treps, C. Fabre, Phys. Rev. A 71, 022313 (2005)

[7] A. Heidmann, R.J. Horowicz, S. Reynaud, E. Giacobino, C. Fabre, G. Camy, Phys. Rev. Lett. 59, 2555 (1987); J. Mertz, T. Debuisschert, A. Heidmann, C. Fabre, E. Giacobino, Opt. Lett. 16, 1234 (1991); J. Gao, F. Cui, C. Xue, C. Xie, K. Peng, Opt. Lett. 23, 870 (1998)

[8] P. Grangier, J.-M Courty, S. Reynaud Opt. Commun. 89, 99 (1992)

[9] L.-M. Duan, G. Giedke, J. I. Cirac, P. Zoller, Phys. Rev. Lett. 84, 2722 (2000)

[10] G. Giedke, M.M. Wolf, O. Krüger, R.F. Werner, J.I. Cirac, Phys. Rev. Lett 91, 107901 (2003)

[11] G. Vidal, R.F. Werner, Phys. Rev. A 65, 032314 (2002)

[12] A. Einstein, B. Podolsky, N. Rosen, Phys. Rev. 47, 777
(1935)

[13] M. Reid, P. Drummond, Phys. Rev. Lett. 60, 2731 (1989); M. Reid, Phys. Rev. A 40, 913 (1989); M. Reid, P. Drummond, Phys. Rev A 41, 3930 (1991)

[14] Z.Y. Ou, S.F. Pereira, H.J. Kimble, K.C. Peng, Phys. Rev. Lett. 68, 3663 (1992)

[15] K. McKenzie, N. Grosse, W.P. Bowen, S.E. Whitcomb, M.B. Gray, D.E. McClelland, P.K. Lam, Phys. Rev. Lett 93, 161105 (2004)

[16] E.J. Mason, N.C. Wong, Opt. Lett. 23, 1733 (1998)

[17] C. Fabre, E.J. Mason, N.C Wong, Optics Communications 170, 299 (1999)

[18] A. Pikovsky, M. Rosenblum, J. Kurths, Synchronization (Cambridge University Press, 2001)

[19] L. Longchambon, J. Laurat, T. Coudreau, C. Fabre, Eur. Phys. J. D 30, 279 (2004)

[20] L. Longchambon, J. Laurat, T. Coudreau, C. Fabre, Eur. Phys. J. D 30, 287 (2004)

[21] N. Korolkova, G. Leuchs, R. Loudon, T.C. Ralph, C. Silberhorn, Phys. Rev. A 65, 052306 (2002)

[22] W.P. Bowen, R. Schnabel, H.-A. Bachor, P.K. Lam, Phys. Rev. Lett. 88, 093601 (2002)

[23] V. Josse, A. Dantan, L. Vernac, A. Bramati, M. Pinard, E. Giacobino, Phys. Rev. Lett. 91, 103601 (2003) 
[24] J. Hald, J.L. Sørensen, C. Schori, E.S. Polzik, Phys. Rev. Lett. 83, 1319 (1999)

[25] M.M. Wolf, J. Eisert, M.B. Plenio, Phys. Rev. Lett 90, 047904 (2003)
[26] G. Adesso, A. Serafini, F. Illuminati, Phys. Rev. A 70, 022318 (2004) 